\title{
Collision-free Time Slot Reuse in Multi-hop Wireless Sensor Networks
}

\author{
\# Lodewijk van Hoesel, Paul Havinga \\ Department of Electrical Engineering, Computer Science and Mathematics, \\ University of Twente \\ Postbus 217, NL-7500 AE Enschede, The Netherlands, \\ E-mail: \{lodewijk.vanhoesel, paul.havinga\}@utwente.nl
}

\begin{abstract}
To ensure a long-lived network of wireless communicating sensors, we are in need of a medium access control protocol that is able to prevent energy-wasting effects like idle listening, hidden terminal problem or collision of packets. Schedulebased medium access protocols are in general robust against these effects, but require a mechanism to establish a nonconflicting schedule. In this paper, we present such a mechanism which allows wireless sensors to choose a time interval for transmission, which is not interfering or causing collisions with other transmissions. In our solution, we do not assume any hierarchical organization in the network and all operation is localized.

We empirically show that our localized algorithm is successful within a factor 2 of the minimum necessary time slots in random networks; well in range of the expected (worst case) factor 3-approximation of known first-fit algorithms. Our algorithm assures similar minimum distance between simultaneous transmissions as CSMA(/CD)-based approaches.
\end{abstract}

\section{INTRODUCTION}

The network diameter of wireless sensor networks (WSNs) is expected to be larger than the transmission and interference ranges of the individual wireless sensors. WSNs are thus assumed to be multi-hop networks, which allows for spatial reuse of the wireless medium. Obviously, this is beneficial for the network, because more data can be transported per second per meter (i.e. higher transport capacity) [3], but it also requires the medium access control (MAC) protocol to take measures for ensuring successful transmissions and to prevent problems like the well-known hidden terminal problem (Section 2). Here is tacitly assumed that all devices operate at identical radio channels, but this is a common assumption in the research field of WSNs.

The idea of multi-hop communication originates from the 1990's (Section 2) and is eagerly incorporated in the WSN research field, because a tremendous amount of energy can be saved by letting nodes in the network assist each other in forwarding packets. Until now, there are only a few cases known that demonstrated multi-hop communication in WSNs and all with a small network diameter. An example is described in [16], where 98 nodes were deployed in a small multi-hop network to monitor the habitat of birds.

In [18], we presented the LMAC protocol that is fit to function in a multi-hop, energy-constrained wireless sensor network. It targets especially energy-efficiency, self-configuration and distributed operation. The LMAC protocol is based upon scheduled access. Each node gets periodically a time interval in which it is allowed to control the wireless medium according its own requirements and needs. Outside this interval, nodes are notified when they are intended receivers. When a node is not needed for communication, it switches its transceiver to standby and is hence able to conserve energy. Since each node gets its own turn in using the medium, there will be little collision of messages which is in other types of MAC methods - such as carrier sense multiple access (CSMA)- one of the main reasons for energy waste [19]. The LMAC protocol is shortly discussed in Section 3.

In this document, we present a lightweight and localized algorithm (Section 5) that allows wireless nodes to choose a time slot, which is not interfering with other transmissions. Our algorithm does not rely on central managers or hierarchical relations in the network - like in [15] and [14]- and adding of new nodes does not require the entire network to reconsider the schedule. Price that has to be paid for this self-configuring is that collisions can occur when many nodes become active at once. This happens only during network setup or in very dynamic topologies, where many nodes are mobile. The LMAC protocol includes an efficient collision resolution mechanism, that is able to resolve collisions [18].

As stated in [11] and [15], the problem of assigning time slots to nodes (i.e. creating a schedule) is related to the well-know (NP-hard) graph coloring problem. The approach chosen in the LMAC protocol assumes that the schedule in the network is fixed (with the exception that nodes reconsider their time slot choice when they interfere with other nodes) and is repeated periodically. The goal is to create a feasible schedule that gives every node the opportunity to communicate with its neighbors. To do this, a certain number of time slots is necessary. The number of time slots to use in the network depends heavily on local maximum network connectivity $\Delta$. In Section 4, we determine theoretical bounds on the number 
of time slots necessary.

In Section 6, we empirically determine the performance of our distributed time slot assignment algorithm and Section 7 summarizes the conclusions of this paper.

\section{RELATED WORK}

\section{A. From single-hop to multi-hop}

In [1] and [2] are the first ideas for random medium access protocol and packet broadcasting described. The ALOHA protocol is developed in the late 1960's at the university of Hawaii in order to facilitate communication between computer terminals. These terminals were located on separated islands of Hawaii. Wireless communication was therefore a convenient manner of communication.

Communication systems up to then were point-to-point connections which consisted of continuous transmission of data. Key decision in the project was "to transmit user information in a single high speed packet burst" and to broadcast the information to multiple receivers [2]. This concept created the need of some form of sharing a common communication channel resource.

The chosen communication channel sharing method is simple. When a terminal has any data to send, it just sends the information at that moment. When no other node is transmitting a packet at the same time, the transmission succeeds ${ }^{1}$. In the case that another terminal is already using the channel, both transmitting terminals detect this and schedule a retry of transmission after a random period. The price to be paid for simplicity in this protocol, is its poor use of the channel capacity; the maximum throughput of the ALOHA protocol is only $18 \%$ [1].

However, a modification to the protocol can increase the channel utilization considerably. In slotted ALOHA time is divided into slots, and nodes may only start transmitting at the beginning of a slot. This organization halves the probability of a collision and raises the channel utilization to around $35 \%$ [8]. Note that in this MAC protocol design, energy-usage is of no importance. Also, the protocol functions only when all terminals are in reach of each other (i.e. no multi-hop communication).

Carrier sense multiple access (CSMA) -originating of the mid 1970's- is an extension of the above described protocol ALOHA. In the protocol, communication between nodes is not scheduled in a strict sense, but nodes keep track of the usage of the wireless channel before they start transmitting [9]. If the medium is in use, nodes postpone their transmissions until the channel is considered to be free again.

This mechanism greatly improves the channel utilization in a one-hop network compared to pure ALOHA or slotted ALOHA: $80 \%$ [9].

In a multi-hop network however, the CSMA protocol suffers from the hidden terminal problem. When two transmitters are

\footnotetext{
${ }^{1}$ The terminals are able to detect wether their transmission was successful (i.e. the intended receiver was able to successfully extract the information in the transmission burst). This is key-issue in the protocol.
}

out of range of each other, they are not able to sense each others transmission, but still their messages might collide at a receiver which is in range of both transmitters. This problem has a large (negative) impact on the channel utilization and was first described in [7]. To improve the performance of the protocol in a multi-hop environment, Karn proposes a handshaking mechanism (known as MACA protocol [7]) which requires a transmitting terminal $T$ first to announce its transmission to the receiver $R$ with a request to send (RTS) message. The receiver $R$ should then answer with a clear to send (CTS) message. This CTS message can be overheard by a transmitter $Z$ assessing the channel and thus $Z$ knows that the channel is busy and schedules a re-attempt for transmitting its message. The transmitter $T$ sends its data to receiver $R$, when it has successfully received the CTS message of $R$. The RTS and CTS messages are typically very short messages and thus they do not have a great negative impact on the channel utilization.

An extension to the MACA protocol is the MACAW [4] protocol; after the data burst of $T$, receiver $R$ responds with an additional message, the acknowledgement (ACK) message. Transmitter $T$ knows at that stage that its data message is correctly received. When $T$ does not receive an ACK message, it re-schedules a new attempt to resend the message after a random time interval. The concept of a four-way handshake is generally known as carrier sense multiple access with collision avoidance (CSMA/CA). Most of the MAC protocols used in wireless sensor networks are based on this principle. Well known examples are SMAC [19] and TMAC [17].

\section{B. MAC protocols for WSNs}

Although the research field of wireless sensor networks is relatively new, many interesting medium access control protocols, designed for this type of networks, have been proposed in literature. In this section, we limit ourselves to introducing the design goals and the methods of spatial medium reuse of MAC protocols for WSNs.

1) Goals: Since the sensors, equipped with RF transceivers and processing units - the so-called sensor nodes-, are typically powered by batteries, one of the most interesting challenges is to make long-lived, ad-hoc and wireless networks of sensors.

In the recent state of technology, the RF transceiver consumes most energy in the sensor node architecture [8]. Lowpower transceivers, suitable for this architecture, consume typically in receiving or transmitting state in the order of $30 \mathrm{~mW}$ and in standby state less than $8 \mu W$ [12]. It is key issue in MAC protocols for WSNs that the transceiver is switched to standby state, whenever possible, to ensure a reasonable battery lifetime.

MAC protocols for ad-hoc wireless networks - like in WLAN or PAN standards- assume plenty of resources available and are therefore ill-suited for WSNs, simply because these resources are not available in the cheap wireless sensors. A $3 W h$ battery is already reasonable sized for a wireless sensor and has to last for over a couple of years. For comparison: 


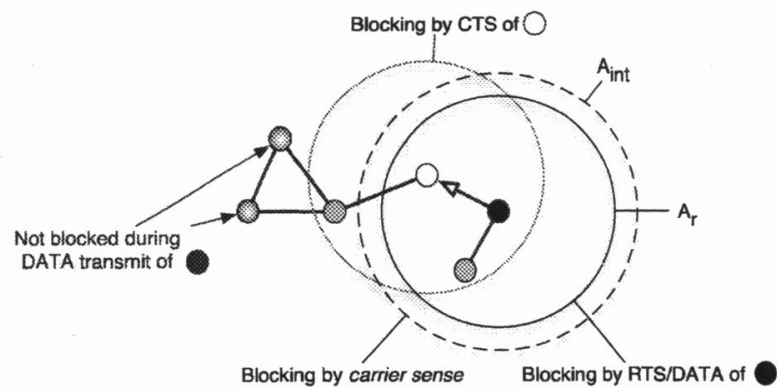

Fig. 1: Transmitter and receiver pair prevents the medium being used during their data transfer

a laptop has a typical battery capacity of $60 \mathrm{Wh}$, which has to last for a only few hours. It is clear that a transceiver consuming several tenths of $\mathrm{mW}$ 's does not significantly have an effect the on-time of a laptop and hence WLAN or PAN standards are not designed with battery-lifetime first and foremost in mind.

Note that efficient use of the transceiver has a large impact on battery lifetime - as we argued-, but has little impact on the volume of data that can be transmitted or received, due to the large energy consumption differences of the transceiver modes. We conclude that efficient networking protocols are required, which are especially tailored for the class of devices in WSNs. The main goal in designing MAC protocols for WSNs is to minimize energy waste - due to collisions of messages and idle listening-, while limiting latency and loss of data throughput [19], [17], [10], [13], [18], [14], [15].

2) Medium reuse in MAC protocols for WSNs: Medium access control protocols for wireless sensor networks can be divided into two classes: CSMA(/CD)-based and schedulebased. Protocols like SMAC [19] and TMAC [17] rely on the handshaking mechanism and carrier sensing to ensure reuse of the medium outside the range of an active transmitter and receiver pair. During transmission, both transmitting and receiving node block other nodes in their surrounding from engaging data packet exchange (Figure 1), but the wireless medium can freely be reused outside this blocking area.

TRAMA [14] is a schedule-based protocol, where nodes compete in establishing a schedule. The first phase of the protocol is a random-access phase in which nodes discover their neighbors and establish the schedule. New nodes can enter the network only during this phase. Next, the nodes enter a phase in which time is divided in small time slots and the schedule is more or less fixed. When the schedule is completed, the nodes fall back to the random-access phase. The protocol ensures a distance of three hops or more between concurrent transmissions.

In [15], an algorithm is presented for assigning time slots in a multi-hop network. The so called MMF-TDMA algorithm runs distritbuted, jet parent nodes coordinate the assigning process of their siblings. Once established, the schedule remains fixed. The authors argue that the performance of their Greedy-algorithm for assigning time slots is not resulting
TABle 1: Spatial MEdiUm ReUSE IN WSN MAC PROTOCOLS

\begin{tabular}{|c|c|c|c|}
\hline Protactis & $\min T / 2 v=12$ & $\min$ & 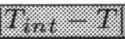 \\
\hline SMAC [19] & $r$ & & $r_{\text {int }}$ \\
\hline TMAC [17] & $r$ & & $r_{i n t}$ \\
\hline DMAC [10] & 0 & & 0 \\
\hline TRAMA [14] & $>r$ & & $>r$ \\
\hline MMF-TDMA [15] & $>r$ & & $>r$ \\
\hline Analysis in [3] & $r_{\text {int }}$ & $\frac{1}{2}($ & $\left.r_{i n t}-r\right)$ \\
\hline
\end{tabular}

necessarily in an optimal solution, but in their simulations the results were empirically found to be optimal. The algorithm ensures that concurrent transmissions can occur only at three hops or more. When a node is added to the network, the algorithm might need to be rerun in order to assure that the new node is assigned a time slot.

In [10] a different method is chosen for assigning time intervals to sensor nodes. Main goal in the DMAC protocol [10] is to reduce latency of data that is designated for the central point in the network and therefore the assignment of the time intervals is dependant on the hop-distance of the node to the central point. Within its time interval a node is assumed to transmit data to a node that is one hop closer to the central point and one time interval before its own a node is expected to listen to the medium and to accept data transfers. The DMAC protocol schedules the medium in very granularly manner; within a time interval, messages of nodes located as equal hop-distance to the central point can still collide. This makes this protocol only suitable for very low data-rates.

In [6] and [3] a model for successful communication is presented. The authors use the notion of transmission/reception range $r$ and interference range $r_{\text {int }}$ to calculate the transport capacity of a wireless multi-hop network. The interference range $r_{\text {int }}$ is typically larger than the transmission/reception range $r$ and communication is said to be successful if there is only one active transmitter in range of a receiver and there is no other active transmitter within interference range. This creates virtual exclusion regions around transmitter/receiver pairs.

In Table 1 we give a summary of the discussed approaches to spatial reuse of the wireless channel. We use min $\left|T_{\text {int }}-R\right|$ to indicate the minimum distance between the receiver $R$ and an potentially interfering transmitter $T_{\text {int }}$ allowed in the MAC protocol. We conclude that we need to have at least a distance of three hops ${ }^{2}$ between concurrent transmissions (this assures $\min \left|T_{\text {int }}-R\right|>r$ and that a receiver has only one active transmitter in range). Experiments should be carried out to find out whether this distance really suffices in real-life multihop networks.

\section{THE LIGHTWEIGHT MEDIUM ACCESS PROTOCOL (LMAC) FOR WSNs}

In schedule-based MAC protocols, time is organized in time slots, which are grouped into frames. Each frame has a fixed

\footnotetext{
${ }^{2}$ Note that hop distance $h$ has little relation to the actual distance between nodes and thus the following is not true: $(h-1) r<\left|T_{\text {int }}-T\right| \leq h r$. A hop distance $h>1$ only assures $\left|T_{\text {int }}-T\right|>r$
} 


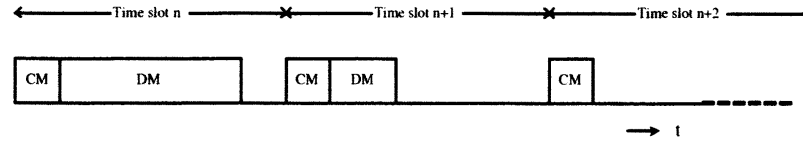

Fig. 2: Time slot contents of the LMAC protocol. The data message (DM) does not have a fixed length and is even omitted when a node does not have any message to send

length of a (integer) number of time slots. The number of time slots in a frame should be adapted to the expected network node density (Section 4 ) or system requirements.

The scheduling principle in the LMAC protocol [18] is very simple: every node gets to control one time slot in every frame to carry out its transmission. When a node has some data to transmit, it waits until its time slot comes up, addresses a neighboring node (or multiple) and transmits the packet without causing collision or interference to other transmissions.

In order to be capable of receiving messages, other nodes always listen at the beginning of time slots of other nodes to find out whether they are addressed either by node ID or by broadcast address.

In the LMAC protocol, a time slot is divided into two parts of unequal length (Figure 2): control message (CM) and data message (DM). A node always starts its time slot by sending out a CM, even if it does not have any data to send. Besides addressing other nodes, the $\mathrm{CM}$ is also necessary for synchronization, resolving collisions and the operation of the distributed time slot scheduling presented in this paper (Section 5).

\section{MAXIMUM NETWORK CONNECTIVITY AND THE NUMBER OF TIME SLOTS}

The problem of assigning time slots in a network is analogous to the NP-hard graph coloring problem. In the time slot assignment, second order neighbors have to be taken in consideration to reuse time slots not sooner than at three hops or more. This is generally known as $E^{2}$-coloring.

Consider a network graph $G$ with nodes $V$ and the connections between the nodes $E$. In such graph we need at least $\Delta+1$ time slots, with maximal connectivity $\Delta=$ $\max \{d(v) \mid v \in V\}$ and $d(v)$ is the number of edges $|E(v)|$ of vertex $v$. This ensures that we can give each node a time slot in the most dense area of the network. Second order nodes have at most $\Delta$ common neighbors with the node with highest connectivity (but are out of reach of that node). If there are $\Delta$ common neighbors, we have to add time slots, but if there are less than $\Delta$ neighbors, time slots might be reused. This illustrates that $\Delta+1$ is indeed a lower bound on the number of time slots.

Note that a neighbor of $v_{i}$ (i.e. node with highest degree) has no more than $\Delta$ neighbors (including $v_{i}$ ):

$$
\zeta\left(v_{i}\right)=\max \left\{d(n) \mid n \in N\left(v_{i}\right)\right\} \leq \Delta
$$

And at most $\zeta$ (with $\zeta-1 \leq \Delta-1$ ) neighbors are second order neighbors to $v_{i}$. In worst case, all second order neighbors can

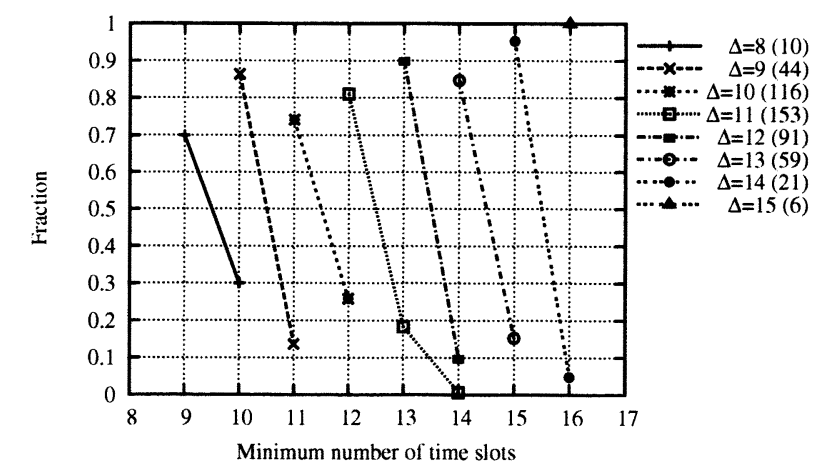

Fig. 3: Number of time slots necessary in random networks for different maximal connectivities (simulation), showing that practical number of time slots is likely to be $O(\Delta)$ instead of $O\left(\Delta^{2}\right)$. Number of topologies in the sets is show between brackets

not reuse time slots and therefore the bounds on the number of time slots are:

$$
\Delta+1 \leq N_{\text {timeslots }} \leq \Delta^{2}-\Delta+1
$$

Note that the upper bound on the number of time slots necessary in a network grows rapidly $\left(O\left(\Delta^{2}\right)\right)$ with increasing maximal connectivity. In practice not all situations will or can occur and therefore practical bounds will be different. We consider 500 connected random deployed networks to empirically determine the number of time slots necessary in this class of graphs. The topologies are generated with average connectivity $2 \pi$ and contain 100 nodes. We divided the the topologies in sets with equal maximum connectivity $\Delta$ and per set the (minimum) number of necessary time slots is determined with extensive search. A certain fraction of the networks within a set could be colored with $\Delta+1, \Delta+2$, etc. colors. These fraction results per set are shown in Figure 3. For example in the set of topologies with $\Delta=8$, in $70 \%$ of the topologies a node-conflicting schedule could be created with 9 time slots.

We conclude from the figure that in these scenarios, the necessary time slots in random deployed networks is likely to be $O(\Delta)$ instead of $O\left(\Delta^{2}\right)$. In worst case, $\Delta+3$ time slots per frame were necessary. More than $70 \%$ of the considered random network topologies could be $E^{2}$-colored with $\Delta+1$ colors.

These are important results for practical reasons. When the number of time slots per frame is large, nodes have to wait long before their time slot comes up to get the opportunity to transmit. This increases the reaction time of the network considerably; an undesired effect. To limit latency, it is key issue to keep the number of time slots in a frame to a minimum.

\section{LOCALIZED ALGORITHM FOR ASSIGNING TIME SLOTS}

In this section, we present a lightweight algorithm that allows nodes to choose a time slot and to be self-configuring in that respect. Main difference with the algorithms presented in [11], 
[14] and [15] is that our algorithm does not rely on central managers nor on hierarchical relations - like leader election or parent/sibling relations- in the network; all operation is localized. We also do not want to use the minimum number of time slots, but we merely want to use a reasonable number, that allows for adding new nodes to the network or for changes in network density due to mobility. In the LMAC protocol, we assume the number of time slots in a frame fixed ${ }^{3}$ and known by all nodes. Let $M$ be the set of time slots.

We define four operational states:

- Initialization state $(I)$ - The node samples the wireless medium (at a low rate to conserve energy) to detect other nodes. When a neighboring node is detected, the node synchronizes (i.e. the node knows the current slot number). When a new frame is due, the node switches to the wait state $W$.

- Wait state $(W)$ - We observed that especially at network setup, many nodes receive an impulse to synchronize at the same time. We introduce randomness in reaction time $W$ between synchronization with the network and the actual choosing of a free time slot: $W=\left\{1, \ldots, W_{\max }\right\}$, expressed in (integer number of) MAC frames. After the random wait time, the node continues with the discover state $D$.

- Discover state $(D)$ - The node collects second order neighborhood information during one entire frame (Section 5-A) and records time slots to be occupied when the signal level is higher that a pre-defined threshold. If all information is collected, the node chooses a time slot (Section 5-B) and advances to the active state $A$. The algorithm of this state is given in Algorithm 1.

- Active state $(A)$ - The node transmits a CM (and DM if necessary) in its own time slot. Meanwhile it listens to other time slots and accepts data from neighboring nodes. The node also keeps its view on the network upto-date. When a neighboring node informs that there was a collision in the time slot of the node (Section 5-C, the node continues in the wait state $W$.

\section{A. Collecting second order neighbor information}

The control message of the LMAC protocol plays an important role for nodes in obtaining a two-hop view of the network. As stated before, this message is always transmitted by nodes in the active state $A$. Every node broadcasts a bit vector of (first order) occupied slots in its CM. In this bit vector, nodes keep track of controlled time slots around them and share this information with neighbors. Each position in the occupied slots bit vector represents a time slot. When a node receives a CM successfully, it updates the vector by setting a logical ' 1 ' at the position of the time slot, otherwise a ' 0 ' will be inserted. A node also inserts a ' 1 ' at the time slot it controls itself.

By simple performing an 'OR'-operation between all received bit vectors, a node in discover state $D$ can determine

\footnotetext{
${ }^{3}$ Although the algorithms in [11] and [15] can be used at network setup to estimate the number of time slots that is necessary and adopt the number of time slots accordingly.
}

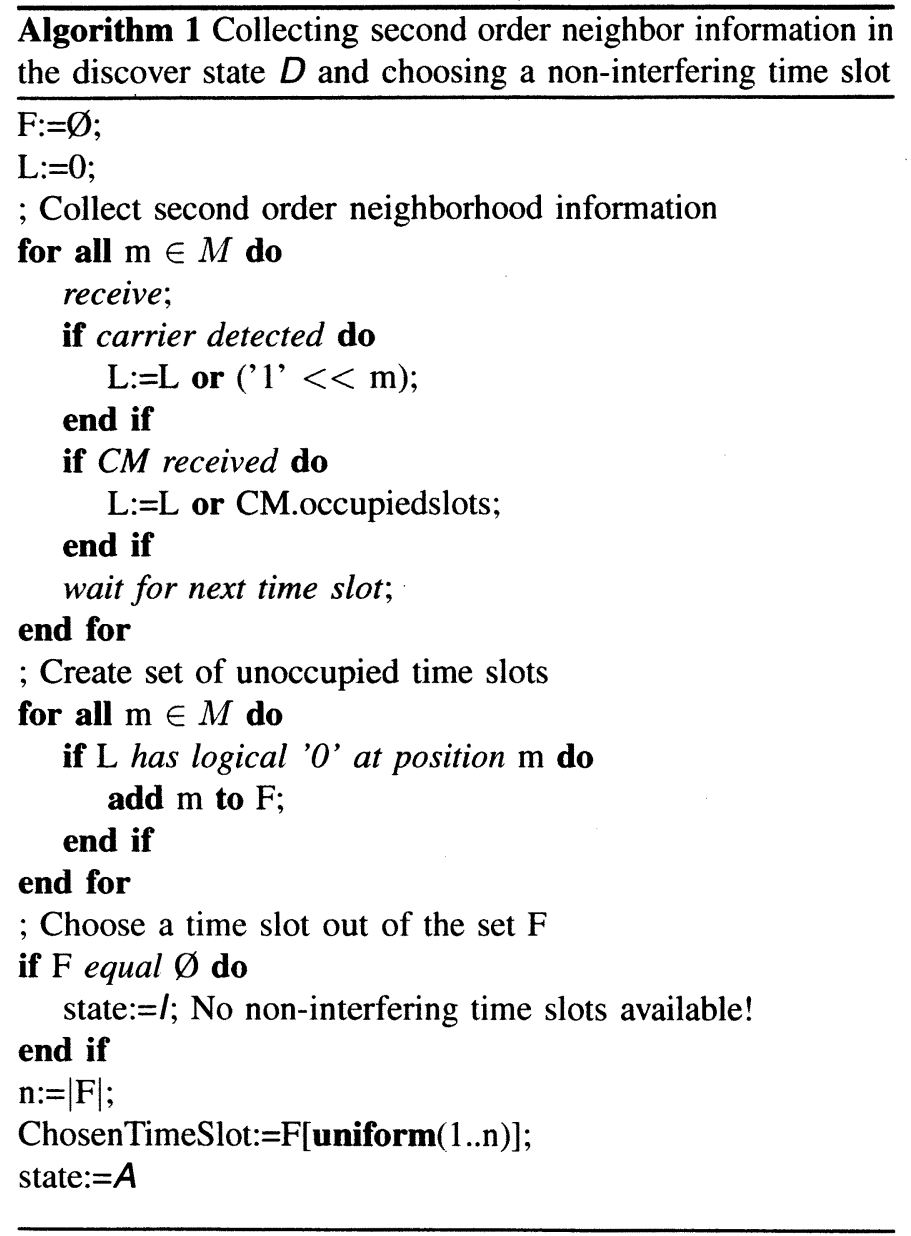

which time slots do not interfere in its second order neighborhood and can be used freely. Let $F$ be the set of non-interfering time slots.

\section{B. Choosing one time slot from the free ones}

At this moment the set $F$ of non-interfering time slots is available. Note that the node can choose any time slot of this set to control. To reduce the probability of collisions (i.e. two or more nodes that claim equal time slots and are interfering with each other), we let nodes randomly choose one from the set $F$.

\section{Resolving collisions}

Collisions can occur when two or more nodes choose the same time slot to control simultaneously. This can happen with small probability at network setup (i.e. many nodes wake-up at same time) or when network topology changes due to mobility of nodes.

The nodes that caused the collision cannot detect the collision by themselves; they need to be informed by their neighboring nodes, simply because they are transmitting when the event occurs. These neighboring nodes use their own time slot to inform the network that they detected a collision by using a special field in the CM of the LMAC protocol. To optimize the inform process, nodes prevent multiple notifications to be 


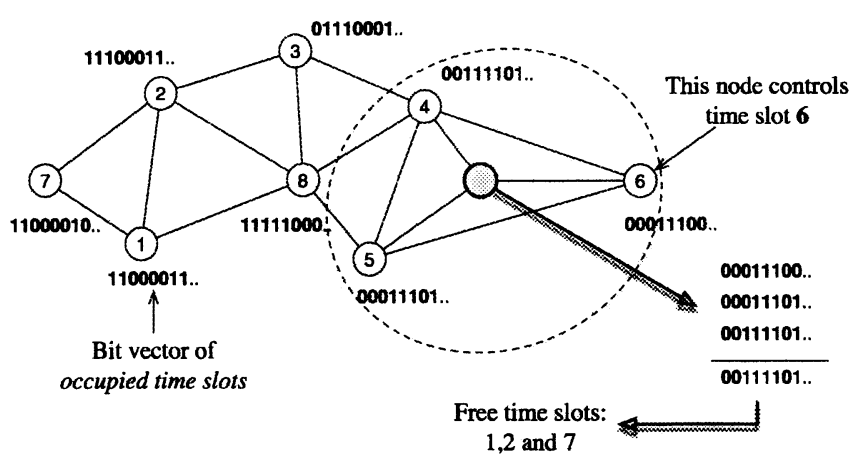

Fig. 4: Illustration of finding free time slots in the discover state

send. This greatly increases the number of colliding time slots that can be reported during a frame. When a node is informed that its CM and - possibly - its DM collided, it will give up its time slot and fall back to the wait state $W$.

\section{Minimum time slot reuse distance in LMAC}

It is simple to see that time slots are only reused after three hops or more (Figure 4). One could see the CM of a node as RTS message, while the acknowledgement of reception of another node in its occupied slots field, can be seen as CTS message. By also indicating a time slot occupied when a carries is detected, our algorithm assures thus a similar distance $\min \left|T_{\text {int }}-T\right|>r_{\text {int }}$ as SMAC or TMAC (Section 2). The minimum distance between receiver $R$ and interfering transmitter $T_{\text {int }}$ is $\min \left|T_{\text {int }}-R\right|>r$.

\section{Performance of the algorithm}

In literature, many studies have been made to find heuristics that are able to find the minimum number of colors for given graphs. Since graph coloring is an NP-hard problem, heuristics trade in general accuracy for reduction of execution time and it is shown that first-fit techniques like Greedy-approaches, approximate the minimum amount of colors by a factor 3 (in worst case) [5]. Our approach is as well based on the first-fit (Greedy-approach) heuristic (yet we try to spread out the slot choice to minimize the number of collisions during rollout of the network). We therefore expect our algorithm to perform in the same order.

By simulation, we verify the performance of the algorithm. The same network 500 network topologies are used as in Section 4. Node with ID ' 0 ' is assigned time slot ' 1 ' and is put into the active state $A$. Other nodes are put in the initialization state $l$. We vary the number of time slots used by the algorithm and record in which topologies non-interfering time slots could be successfully assigned to all nodes. To rule out effects of the randomness in the algorithm, we record only runs to be successful if for ten different random seeds all nodes could choose non-interfering time slots. In Figure 5 the results are shown. Again, we divided the topologies in sets with equal maximum connectivities and plotted the fraction of a set that could be colored with a specific number of time slots. We

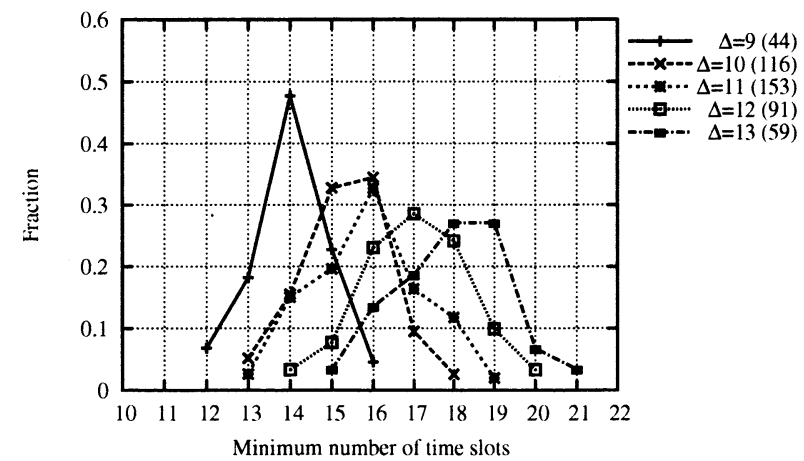

Fig. 5: Number of time slots necessary in random networks for different maximal connectivities using the localized algorithm of Section 5 (simulation)

conclude that the localized algorithm is successful within a factor 2 of the minimum necessary time slots; well in range of the expected performance.

\section{Conclusion}

When nodes are separated far enough in a multi-hop network, they can use the wireless medium for transmitting simultaneously, without causing collision or significant interference. This reuse of the wireless medium is beneficial for the network, because it increases the transport capacity and it can -if nodes assist each other in forwarding data- potentially reduce the energy required for transmission. We studied the medium reuse for a schedule-based MAC protocol.

We determined theoretical bounds for the number of time slots necessary in a schedule, to give every node opportunity to communicate collision-free. This scheduling problem is related to the well-known graph coloring problem and we found that the minimum number of time slots necessary is dependant on the maximum connectivity: $\Delta+1 \leq N_{\text {timeslots }} \leq \Delta^{2}-$ $\Delta+1$. The upper bound grows fast (quadratic) with increasing maximum connectivity. By experimentation we discovered that practical bounds tend to be equal to the lower bound $(70 \%$ of the cases) or very close to it (within two additional time slots). Therefore the number of time slots in frame can be kept reasonably small. These are important results for practical reasons. When the number of time slots per frame is large, nodes have to wait long before their time slot comes up to get the opportunity to transmit. This increases the reaction time of the network considerably; an undesired effect. To limit latency, it is key issue to keep the number of time slots in a frame to a minimum.

In this paper, we presented a lightweight and localized time slot assignment algorithm, which allows nodes to be self-configuring in that aspect. We presented a mechanism that allows nodes to discover which time slots can be used without interfering with other nodes in their second order neighborhood or causing collisions. Our method does not rely on central managers or hierarchical relations in the network and the addition of new nodes does not require the entire network to reconsider the schedule. Our algorithm assures similar minimum medium reuse distances as CSMA(/CD) 
based approaches. We concluded that our localized algorithm is successful within a factor 2 of the minimum necessary time slots; well in range of the expected performance.

\section{REFERENCES}

[1] N. Abramson. The aloha system- another alternative for computer communications. Proc. 1970 Fall Joint Computer Conf., vol. 37:281$285,1970$.

[2] N. Abramson. Development of the alohanet. IEEE Transactions on information theory, vol. 31 no. 2:119-123, March 1985.

[3] A. Agarwal and P.R. Kumar. Improved capacity bounds for wireless networks. Wireless Communication and Mobile Computing, vol. 4:251261 , July 2004.

[4] V. Bharghavan, A. Demers, S. Shenker, and L. Zhang. Macaw: a media access protocol for wireless lans. In Conf. on Communications Architectures, Protocols and Applications, pages 212-225, Augustus 1994.

[5] Jiří Fiala, Aleksei V. Fishkin, and Fedor V. Fomin. On distance constrained labeling of disk graphs. Theoretical Computer Science, 326(1-3):261-292, 2004.

[6] P. Gupta and P.R. Kumar. The capacity of wireless networks. IEEE Transactions on Information Theory, vol. IT-46 no. 2:388-404, March 2000.

[7] P. Karn. Maca - a new channel access method for packet radio. In 9th ARRL Computing Networking Conference, pages 134-140, September 1990.

[8] K.Langendoen and G. Halkes. The Embedded Systems Handbook. CRC Press, To be published . http://www.st.ewi.tudelft.n1/ koen/papers/MAC-chapter.pdf.

[9] L. Kleinrock and F.A. Tobagi. Packet switching in radio channels: Part 1-carrier sense multiple-access modes and their throughput-delay characteristics. IEEE Transactions on Communications, Vol. 23, No. 12, December 1975.

[10] G. Lu, B. Krishnamachari, and C.S. Raghavendra. An adaptive energyefficient and low-latency mac for data gathering sensor networks.

[11] T. Moscibroda and R. Wattenhofer. Coloring unstructured radio networks. 17th Symposium on Parallelism in Algorithms and Architectures, July 2005.

[12] Nordic. nrf905 datasheet. http://www.nordicsemi.no.

[13] J. Polastre, Jason Hill, and David Culler. Versatile low power media access for wireless sensor networks. Proceedings of the Second International Conference on Embedded Networked Sensor Systems (SenSys), pages 95-107, November 2004.

[14] V. Rajendran, K. Obrazka, and J. Garcia-Luna-Aceves. Energy-effcient, collision-free medium access control for wireless sensor netwoks. Conference on Embedded Networked Sensor System, pages 181-192, 2003.

[15] A. Sridharan and B. Krishnamachari. Max-min fair collision-free scheduling for wireless sensor networks. Workshop on multi-hop wireless networks, 2004.

[16] R. Szewczyk, A. Mainwaring, J. Polastre, J. Anderson, and D. Culler. An analysis of a large scale habitat monitoring application. Second international conference on embedded networked sensor systems (SENSYS), pages 214-226, November 2004.

[17] T. van Dam and K. Langendoen. An adaptive energy-efficient mac protocol for wireless sensor networks. In Ist ACM Conf. on Embedded Networked Sensor Systems (SenSys 2003), page 171180, November 2003.

[18] L.F.W. van Hoesel and P.J.M. Havinga. A lightweight medium access protocol (lmac) for wireless sensor networks: Reducing preamble transmissions and transceiver state switches. INSS, June 2004.

[19] W. Ye, J. Heidemann, and D. Estrin. An energy-efficient mac protocol for wireless sensor networks. Twenty-First Annual Joint Conference of the IEEE Computer and Communications Societies (INFOCOM), Vol. 3:1567-1576, June 2002. 